\title{
OFDM over IEEE 802.11b hardware for telemedical applications
}

\author{
Konstantinos A. Banitsas, ${ }^{*}$ Yong Hua Song \\ and Thomas J. Owens
}

Department of Electronic \& Computer Engineering, Brunel University, Uxbridge, UB8 3PH, UK

Fax: +441895258728 E-mail: bany@hol.gr

E-mail: y.h.song@brunel.ac.uk E-mail: thomas.owens@brunel.ac.uk

${ }^{*}$ Corresponding author

\begin{abstract}
Using a wireless Local Area Network (WLAN) to transmit live high-quality video suitable for a telemedical application presents many challenges, including ensuring sufficient Quality of Service (QoS) for the end-user to be able to make an accurate diagnosis. One of the many problems that exist when developing such a system is the multipath effect caused by the reflections of the transmitted signals on various surfaces including walls, floors, furniture and people. This degrades the signal quality and reduces the amount of available bandwidth and, thus, the quality of the image. Presently, most of Europe is using the IEEE $802.11 \mathrm{~b}$ hardware for such applications. As an alternative to the existing modulation of $802.11 \mathrm{~b}$, Orthogonal Frequency Division Multiplexing (OFDM) is investigated, especially for use inside hospitals. The advantages of using this modulation over IEEE $802.11 \mathrm{~b}$ hardware for a telemedicine application are examined by means of simulation using three different simulation packages.
\end{abstract}

Keywords: OFDM; IEEE 802.11b; telemedicine; multipath; hospital.

Reference to this paper should be made as follows: Banitsas, K.A., Song, Y.H. and Owens, T.J. (xxxx) 'OFDM over IEEE 802.11b hardware for telemedical applications', Int. J. Mobile Communication, Vol. x, No. x, pp.xxx-xxx.

Biographical notes: Konstantinos Banitsas obtained his BSc and MSc degrees from Portsmouth University, UK, in 1994 and 1996, respectively. He was extensively involved in the research areas of digital signal processing and robotics. Currently, he is a Lecturer and a Researcher in the area of Telemedicine at the Department of Electronic and Computer Engineering of Brunel University, West London.

Yong Hua Song received his BEng, MSc and PhD in 1984, 1987 and 1989, respectively, from China. He joined Brunel University in 1997 as Professor of Network Systems at the Department of Electronic and Computer Engineering. Currently, he is Director of Brunel Institute of Network Systems. $\mathrm{He}$ has published four books and over 300 papers mainly in the areas of applications of intelligent and heuristic methods in engineering systems.

Thomas J. Owens obtained his $\mathrm{PhD}$ in Electrical and Electronic Engineering from Strathclyde University in 1986. In 1987, he joined as a Lecturer at what is now the Department of Electronic and Computer Engineering, Brunel University. He is Course Director for the MSc Data Communication Systems in its off-campus and distance learning modes and the MSc Distributed 
Computing Systems Engineering. He is a Chartered Engineer, Chartered Mathematician and Member of the Institute of Teaching and Learning. He was coordinator of the 2002-2003 EU IST project CONFLUENT. He is the author of more than 60 refereed journal and conference papers.

\section{Introduction}

Nowadays, Wireless Local Area Network (WLAN) systems capable of transmitting at high speeds are being developed and installed around the world. They possess a number of advantages ranging from installation flexibility and mobility to increased scalability.

Hospitals are in need of such technology as it will ensure faster and more accurate addressing of patient needs allowing for delivery of services to the point of care, even though the treating staff could be in a different place at the time. Presently, a joint research project, 'MedLAN', is seeking to address this need [1].

As with any development work, several problems have to be solved before a WLAN system operates successfully. The problems are mostly concerned with the range, speed, security and interference that the wireless system will have [2,3]. Especially on the range issue, there are several factors that can influence the transmission and reception of the signal and affect the overall quality of the application. In contrast with wired systems that have no range problems (apart from the ones dictated by the protocols used which are always predictable), WLANs suffer from a lot of factors that can influence their active range, with the operating terrain being one of the most important. Wireless product vendors acknowledge this fact and try to implement as many techniques as possible to ensure that the signal will eventually be correctly transmitted.

The most widely used wireless protocol today is the IEEE $802.11 \mathrm{~b}$. In the market since 2000, it usually uses Complementary Code Keying (CCK) and Direct Signal Spread Spectrum (DSSS) techniques to spread its signal over a frequency range and avoid interference while achieving a top speed of 11 Mbps. As sophisticated as this technique is, it behaves relatively poorly in multipath environments when compared with newer modulation schemes like Orthogonal Frequency Division Multiplexing (OFDM).

In this paper, the advantages of using OFDM over IEEE $802.11 \mathrm{~b}$ hardware will be investigated with emphasis on the benefit that the end-users will enjoy when working with telemedical applications. Consequently, this paper is organised as follows. In Section 2, an overview of existing wireless technologies is presented. Section 3 is a short presentation of the 'MedLAN' project and the medical needs it addresses. In Section 4, the basics of the OFDM are explained along with the advantages and disadvantages of the technique. Section 5 is a discussion of the IEEE $802.11 \mathrm{~b}$ protocol, listing some of its fundamental properties. In Section 6, the motivation for the simulations carried out is provided and the simulation environment described. The results obtained are presented and analysed in Section 7. In Section 8, the conclusions drawn are presented. 


\section{Existing technologies}

Presently, most of Europe is using WLANs based on an IEEE standard, 802.11b. These kinds of WLANs have a top speed of 11 Mbps and an advertised range of about $300 \mathrm{~m}$. However in practice, the maximum available speed is about $2.5 \mathrm{Mbps}$ and their useful range inside an office space is about $20-30 \mathrm{~m}$. This range and speed is even less when the operating terrain consists of many obstacles like thick walls, metallic surfaces, furniture and even people walking within the range of the WLAN [4]. IEEE 802.11b operates in the 2.4 frequency band (a band also known as Industry, Science and Medicine; ISM). The latter is one of the least favourite frequency bands, as at $2.44 \mathrm{GHz}$ water molecules resonate and microwave ovens operate [5]. To cope with this problem, WLANs usually use some kind of spread spectrum technique, either Frequency Hopping Spread Spectrum (FHSS) or DSSS to spread their signal over a frequency range so they can avoid interference at a specific frequency. DSSS is the most widely used method and is considered slightly better than FHSS.

Regardless of the spreading technique used, the wireless transmission signal will still suffer from attenuation, especially when the Mobile Terminal (MT) is moving away from the Access Point (AP). For this reason 802.11b is designed to fall back on its speed in order to maintain signal integrity. This means that as the received signal is deteriorating, the connection speed will automatically fall from $11 \mathrm{Mbps}$ to 5.5, 2 and finally to $1 \mathrm{Mbps}$. Keeping in mind that real-time applications (like telemedicine) are bandwidth demanding, the reduced bandwidth is rarely capable of accommodating the needs of real-time applications.

An attempt to increase the available bandwidth is the IEEE protocol, 802.11a, capable of operating at speeds of as high as $54 \mathrm{Mbps}$. Although suggested and standardised first, 802.11 a was released after 802.11 b and up until recently was only operating in the USA. This is because 802.11 a operates in the $5 \mathrm{GHz}$ band that is reserved in most European countries. This band is called Universal Networking Information Infrastructure (UNII) and consists of three separate bands: UNII-1 for indoor use, UNII-2 for either indoor or outdoor use and UNII-3 for outdoor bridging only (Table 1) [6].

Table 1 The three UNII frequency bands for use with 802.11a and Hiperlan/2

\begin{tabular}{|l|c|c|c|c|c|}
\hline $5 \mathrm{GHz}$ band & $\begin{array}{c}\text { UNII-1: } \\
5.15-5.25 \mathrm{GHz}\end{array}$ & $\begin{array}{c}\text { UNII-2: } \\
5.26-5.35 \mathrm{GHz}\end{array}$ & $5.36-5.47 \mathrm{GHz}$ & $5.471-5.725 \mathrm{GHz}$ & $\begin{array}{c}\text { UNII-3: } \\
5.726-5.825 \mathrm{GHz}\end{array}$ \\
\hline $\begin{array}{l}12 \text { channels } \\
\text { (US) }\end{array}$ & $50 \mathrm{~mW}$ & $250 \mathrm{~mW}$ & Reserved & Reserved & $1 \mathrm{~W}$ \\
\hline $\begin{array}{l}19 \text { channels } \\
\text { (EU) }\end{array}$ & \multicolumn{2}{|c|}{$200 \mathrm{~mW}$} & Reserved & $1 \mathrm{~W}$ & Reserved \\
\hline
\end{tabular}

Recently, a few European countries including UK and The Netherlands deregulated the $5 \mathrm{GHz}$ spectrum.

Although 802.11a seems to answer to the needs of real-time applications the $5 \mathrm{GHz}$ band suffers from reduced range when compared to the $2.4 \mathrm{GHz}$ band as the need for line-of-site is more dominant. Furthermore, reflections are much more apparent in the $5 \mathrm{GHz}$ spectrum, so any kind of reflective surface can have devastating effects on signal quality. The most visible consequence is when trying to cover a space with APs, a lot more are needed if 802.11a is used compared to 802.11b [2,7]. 
Figure 1 shows results of an experiment that took place in Central Middlesex Hospital (CMH) that investigated the number of APs needed to cover the Accidents and Emergency (A\&E) space using both 802.11a and b technologies. Squares indicate APs using 802.11b while circles show APs using IEEE 802.11a. It is clear that when using 802.11a a greater number of APs (seven APs) are required to cover the same space, compared to $802.11 \mathrm{~b}$ (two APs).

Figure 1 Range comparison between 802.11a and 802.11b. Squares are $802.11 \mathrm{~b}$ and circles $802.11 \mathrm{a}$

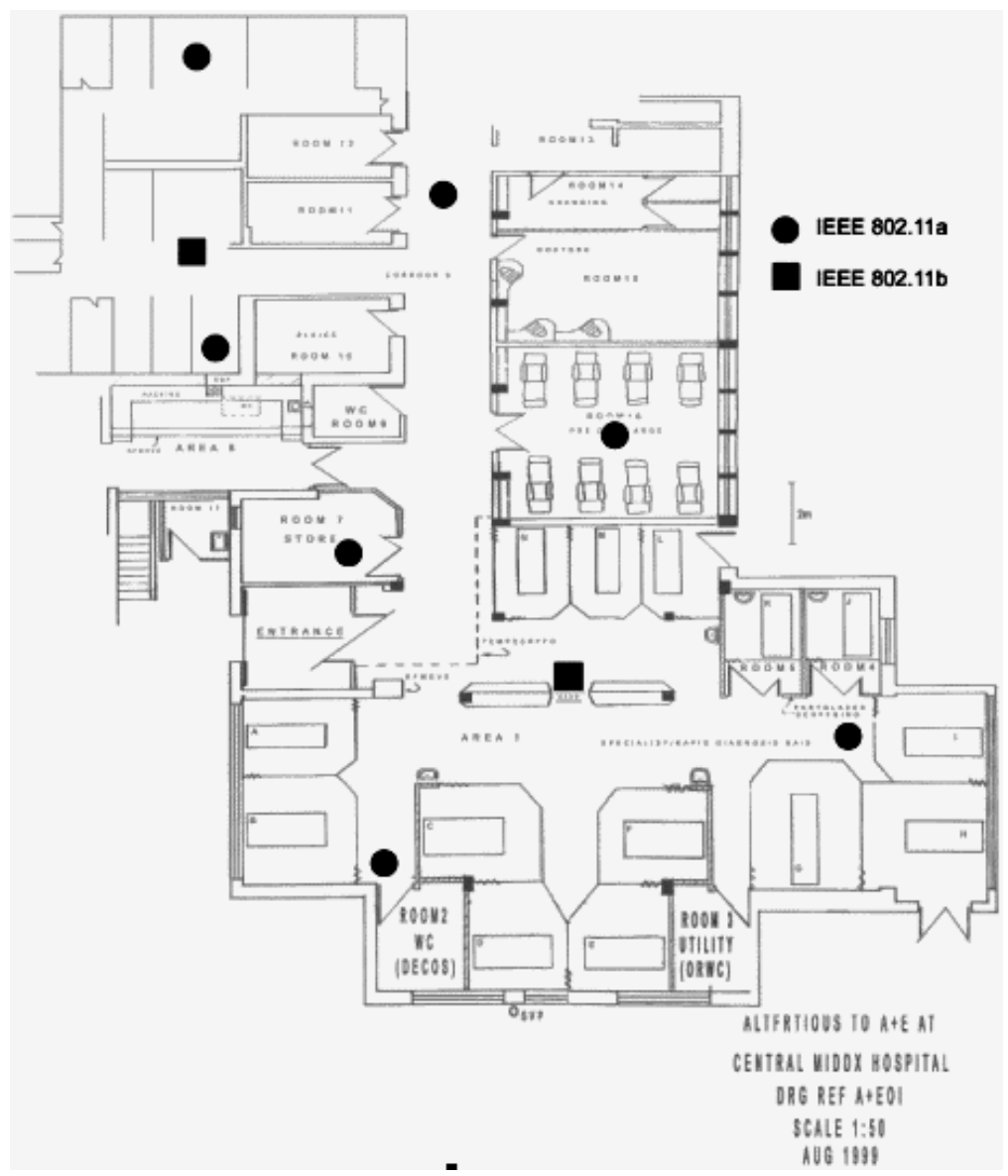

Furthermore, as mentioned above, 802.11a suffers from signal deterioration due to reflecting surfaces. Several studies on the reflection of common materials agree that the reflections in the $5 \mathrm{GHz}$ band are more severe than in the $2.4 \mathrm{GHz}$ band. Table 2 summarises some of these effects in both frequency bands $[4,8]$. 
OFDM over IEEE $802.11 \mathrm{~b}$ hardware for telemedical applications

Table 2 Transmission and reflection values of common materials for ISM and UNII bands

\begin{tabular}{lccccc}
\hline & \multicolumn{3}{c}{$T(d B)$} & & \multicolumn{2}{c}{$R(d B)$} \\
\cline { 2 - 3 } \cline { 5 - 6 } Material & ISM & UNII & & ISM & UNII \\
\hline Dry red brick & -4.43 & -14.62 & & -12.53 & -8.98 \\
Drywall $(12.8 \mathrm{~mm})$ & -0.49 & -0.51 & & -12.11 & -11.50 \\
Drywall $(9 \mathrm{~mm})$ & -0.50 & -0.84 & & -12.03 & -8.87 \\
Body of water & -14.2 & -3.8 & & -10.50 & 8.91 \\
\hline
\end{tabular}

It is clear that since thick red brick walls (often found in hospitals) have a greater reflection index than concrete walls, they create a stronger multipath phenomenon as the operating frequency increases. Note that a body of water simulates the human body, which mainly consists of water and might move in between the communications path. Since the ISM band operates at $2.4 \mathrm{GHz}$, the transmitted energy is absorbed much more by water molecules than in the $5 \mathrm{GHz}$ band. However, reflections due to water obstacles (and thus human bodies) tend to be less of a factor in the ISM band.

Finally, it is worth mentioning that a new protocol, HIgh PERformance Local Area Network (Hiperlan/2), also operating in the $5 \mathrm{GHz}$ band, has been developed by the European Technical Standards Institute (ETSI) and promises true Quality of Service (QoS) and speeds of $54 \mathrm{Mbps}$. Unfortunately, despite its development for over 4 years, hardware supporting this protocol is not yet commercially available.

Hiperlan/2 shares most of the characteristics of 802.11a with the addition of QoS. This means that the system can prioritise traffic into different classes, so real-time applications can enjoy higher priority than standard traffic and reduced delivery times over the air medium $[9,10]$.

Both Hiperlan/2 and 802.11a share almost the same characteristics with the exception of the intermediate fallback speeds: 6, 9, 12, 18, 24, 36, 48, 54 Mbps for IEEE 802.11a and $6,9,12,18,27,36,54$ for Hiperlan/2. Since the modulation is practically the same, one can conclude that the issues discussed in this paper concerning 802.11a apply to Hiperlan/2 [11].

\section{A telemedical application}

Mobile telemedicine is a new and evolving area of telemedicine that exploits the recent developments in mobile networks for telemedical applications in general. Presently, a project named 'MedLAN' is seeking to address some specific medical needs: freedom of movement inside the A\&E area while transmitting high-quality video [1].

MedLAN consists of two main parts: a mobile trolley that exists in the open plan A\&E area and a consultation point, within the hospital.

The mobile trolley consists of a high-end laptop computer that is equipped with a WLAN PCMCIA card using the IEEE $802.11 \mathrm{~b}$ protocol that permits total mobility within the A\&E room and beyond. An AP within the A\&E department acts as a wireless bridge for the network data to be transmitted to and received from the rest of the network. A high-quality digital camcorder is connected to the laptop and high-quality video and audio and still pictures can be transmitted. Medical instruments like otoscopes, dermascopes can also be connected to the system (Figure 2). 
Figure 2 MedLAN can create a telemedical link between the A\&E ward and a consultation point

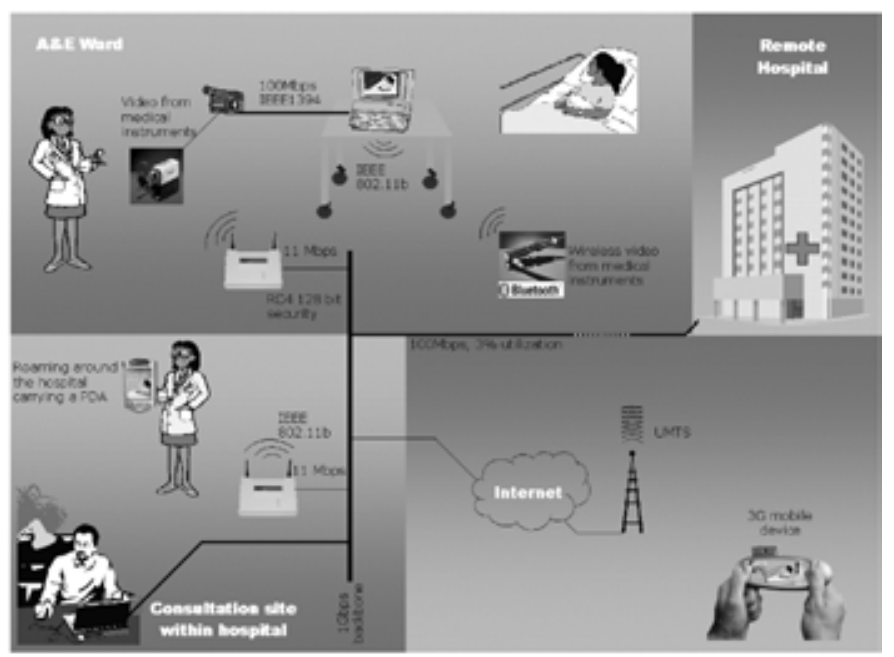

In the consultation point (either within the same hospital or in another NHS hospital), the consulting physician can have a choice of teleconferencing either from a fixed computer within the existing hospital network, or from a mobile computer, sharing the same mobility advantages as the transmitting laptop. It can transmit video to a PDA or even use $3 \mathrm{G}$ mobile devices as terminals $[12,13]$.

It is obvious that in these kinds of systems, QoS plays a critical role. It would be useless if the system could not guarantee the level of service necessary for accurate diagnosis. Despite the fact that none of the IEEE 802.11 protocols have guaranteed QoS, there are several parameters that a developer can optimise in order to keep a high operational level of the wireless network. Some of these include: connection establishment delay, throughput, transit delay, residual error rate, protection, priority and resilience. While protection refers to the security that the system applies to the transmitted data [14], throughput and resilience are definitely some of the most important QoS parameters in a WLAN. In order to maximise the throughput one has to minimise the number of errors that appear in the communication channel. One of these erroneous factors is the multipath phenomenon.

In most wireless communications, the signal does not travel from transmitter to receiver through a straight line. Mountains, buildings, floors, ceilings, furniture and even people reflect the signal depending on the operating frequency. The lower the frequency, the more it can penetrate through objects and not get reflected. The higher the frequency, the more reflections take place and a multipath effect is more dominant.

During the multipath effect the receiver not only receives the signal directly from the transmitter, but also receives all the reflections of that signal. What makes this an undesirable effect is that since the straight-line transmission arrives the earliest, all other packet transmissions arrive with a time delay and collide with next-frame data. Depending on the distance between receiver and transmitter and the number of reflected paths, the signal can be rendered useless, even though its power would be sufficient in the absence of this effect.

The above discussion indicates that IEEE 802.11a would suffer from a much higher multipath loss than $802.11 \mathrm{~b}$ since it is using a higher frequency band. 
However, a different kind of modulation from that used in $802.11 \mathrm{~b}$ is being used in both 802.11a and Hiperlan/2 to address this problem; OFDM.

\section{OFDM}

Although OFDM was proposed as far back as 1950, it is the recent developments in VLSI chips and the increased computational power of Digital Signal Processing (DSP) processors that have made it realisable.

Frequency Division Multiplexing (FDM) systems separate the available bandwidth in a number of intermediate channels. To accommodate hardware imperfection and different distances between transmitter and receiver, FDM introduces a number of guard bands between each of the channels so that signal frames do not overlap each other. Unfortunately, these guard bands can take up to $50 \%$ of the available spectrum and thus reduce the spectrum efficiency.

Much like FDM, OFDM also separates the channel into a number of intermediate frequency channels (approximately 2000-8000 for digital TV and 48 for Hiperlan/2). However, in contrast with FDM, OFDM systems do not have any guard bands. In fact, OFDM overlapping subcarriers is a desirable characteristic. The way this is done is by a careful selection of the frequencies used (subcarriers), so that they are orthogonal to each other (Figure 3). Consequently, subcarriers are easily separated without causing any interference among them [15]. OFDM is modulated by applying changes in a carrier, varying its phase, amplitude or both. Typically, high-level modulation techniques, such as Quadrature Amplitude Modulation (QAM), are employed to distribute the data over the carriers spaced at precise frequencies.

Figure 3 OFDM subcarriers are chosen so that an integral number of cycles fit exactly within a symbol period. Figure produced by the simulation of PHY layer of OFDM using VisSim

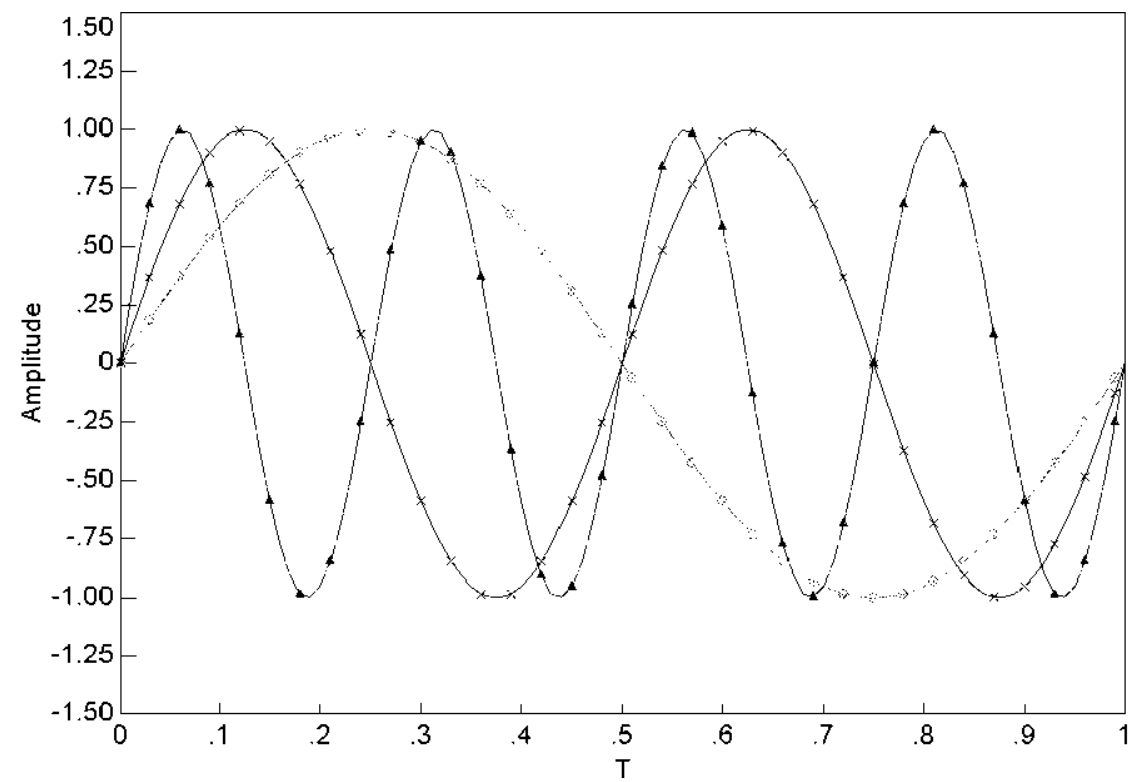


At the implementation level, OFDM systems take a serial data stream and convert it into $N$ parallel data series. Each of these data series is then modulated into a subcarrier at a unique frequency and then the subcarriers are combined to produce a serial stream of transmitted signal. Inverse Fast Fourier Transform (IFFT) is the most common way of realising the above modulation. The decoding procedure is the exact opposite sequence.

Since OFDM transmit data in blocks, multipath delay will cause blocks of signals to collide with each other causing an Inter-Block Interference (IBI) that eventually will lead to Inter-Symbol Interference (ISI) [16]. To address this issue, OFDM uses an additional signal that is added to the beginning of each symbol. This added signal does not contribute any useful information but acts as a guard band to combat channel delay. The most commonly used method of doing the above is the Cyclic Prefix where the last useful part of the signal is copied to the start of the signal. As a result the periodicity of the signal is preserved.

OFDM systems can cope a lot better in multipath environments as data streams are transmitted in parallel and thus require a longer symbol period. For example, in Hiperlan/2, which uses 48 different parallel streams, the symbol period is 48 times as long as if it would be transmitted in a single stream. This, combined with the Cyclic Prefix, considerably improves the tolerance that the signal has against multipath interference: instead of transmitting a very fast single stream, multiple streams are transmitted at a much lower frequency so that interference can only affect a very small portion of the symbol, during its initial part. However, the affected part is only redundant information introduced by the Cyclic Prefix method.

\section{$5 \quad$ Basics of 802.11}

IEEE 802.11 describes the two lower layers of the OSI model: the physical and the data link layer [6]. As described above, it operates in the ISM band and at a frequency of $2.402-2.483 \mathrm{GHz}$ (Europe). Within this frequency band, $802.11 \mathrm{~b}$ creates 11 overlapping channels for communication between APs and client stations. Three of these channels are completely independent. This means that up to three APs can be set to operate in close proximity without interfering with each other. By carefully selecting the channels used and the signal strength, a cell-like topology can be implemented and extended to cover a vast office or campus area. Typical output power dictated by ETSI varies from 30 to $100 \mathrm{~mW}$ for both AP and client cards but can be reduced to $1 \mathrm{~mW}$ by advanced site planning. The maximum nominal speed of $11 \mathrm{Mbps}$ is reduced to 5.5, 2 and finally 1 Mbps as the signal is degraded [17].

Modulation for $802.11 \mathrm{~b}$ depends on the speed. Obligated to be compatible with the 802.11, it supports both FHSS and DSSS. Frequency Hopping solved a lot of early 802.11 problems as the transmitted signal was jumping from one frequency to another so fast $(0.4 \mathrm{~s})$ that the receiver would jump to the next frequency before the reflected signal (due to the multipath effect) could interfere with the main transmission. Unfortunately, FHSS limited the speed to 1 Mbps full duplex (with a much lower practical throughput) $[18,19]$.

802.11b systems running at $1 \mathrm{Mbps}$ use Barker code and BPSK modulation, and those running at 2 Mbps use Barker code and QPSK modulation. Systems running at 5.5 Mbps and 11 Mbps use CCK and QPSK modulation. 
DSSS is the most commonly used spreading technology today. DSSS works by taking a data stream of zeros and ones and modulating it with a second pattern, the chipping sequence. That sequence is known as the Barker code, an 11-bit sequence (10110111000) that has certain mathematical properties making it ideal for modulating radio waves. The basic data stream is XORed with the Barker code to generate a series of data objects called chips. Each bit is 'encoded' by the 11-bit Barker code, and each group of 11 chips encodes 1 bit of data.

Rather than using the Barker code, CCK uses a series of codes called Complementary Sequences. Because there are 64 unique code words that can be used to encode the signal, up to 6 bits can be represented by any one particular code word (instead of the 1 bit represented by a Barker symbol) and thus speed can be as high as $11 \mathrm{Mbps}$.

Finally, a brand new version of wireless networking, IEEE 802.11g, promises speeds as high as $54 \mathrm{Mbps}$, at the ISM frequency band. Unfortunately, in order to maintain compatibility with both $802.11 \mathrm{a}$ and 802.11 b, it was forced to adapt all available modulation schemes, along with Packet Binary Convolution Coding (PBCC), making the hardware considerably more expensive (Table 3). This system is in the process of standardisation; however, hardware has already been commercially available.

Table 3 Comparison table between IEEE 802.11b, a and $\mathrm{g}$ in terms of data rates, carrier and modulations

\begin{tabular}{|c|c|c|c|c|c|c|c|}
\hline \multirow{2}{*}{$\begin{array}{l}\text { Speed } \\
\text { (Mbps) }\end{array}$} & \multirow[b]{2}{*}{ Carrier } & \multicolumn{2}{|c|}{$802.11 \mathrm{~b}$} & \multicolumn{2}{|c|}{$802.11 \mathrm{~g}$} & \multicolumn{2}{|c|}{$802.11 a$} \\
\hline & & Mandatory & Optional & Mandatory & Optional & Mandatory & Optional \\
\hline 1 & Single & Barker & & Barker & & & \\
\hline 2 & Single & Barker & & Barker & & & \\
\hline 5.5 & Single & CCK & $\mathrm{PBCC}$ & CCK & $\mathrm{PBCC}$ & & \\
\hline 6 & Multi & & & OFDM & CCK-OFDM & OFDM & \\
\hline 9 & Multi & & & & $\begin{array}{l}\text { OFDM, } \\
\text { CCK-OFDM }\end{array}$ & & OFDM \\
\hline 11 & Single & CCK & PBCC & $\mathrm{CCK}$ & PBCC & & \\
\hline 12 & Multi & & & OFDM & CCK-OFDM & OFDM & \\
\hline 18 & Multi & & & & $\begin{array}{l}\text { OFDM, } \\
\text { CCK-OFDM }\end{array}$ & & OFDM \\
\hline 22 & Single & & & & $\mathrm{PBCC}$ & & \\
\hline 24 & Multi & & & OFDM & CCK-OFDM & OFDM & \\
\hline 33 & Single & & & & PBCC & & \\
\hline 36 & Multi & & & & $\begin{array}{l}\text { OFDM, } \\
\text { CCK-OFDM }\end{array}$ & & OFDM \\
\hline 48 & Multi & & & & $\begin{array}{l}\text { OFDM, } \\
\text { CCK-OFDM }\end{array}$ & & OFDM \\
\hline 54 & Multi & & & & $\begin{array}{l}\text { OFDM, } \\
\text { CCK-OFDM }\end{array}$ & & OFDM \\
\hline
\end{tabular}


It is evident that $802.11 \mathrm{~b}$ will either support low speeds and consequently have low multipath distortion or operate at a higher speed but suffer from multipath interference.

In the next section, the alternative of using OFDM over $802.11 \mathrm{~b}$ technology will be investigated, with emphasis on the telemedical applications. The idea of OFDM over the $2.4 \mathrm{GHz}$ band is not very new and has been investigated for a number of years and addressed within the IEEE $802.11 \mathrm{~g}$. However, there is very little research both on the use of this modulation in telemedical applications and on the financial advantages of applying OFDM to the existing $802.11 \mathrm{~b}$ systems, instead of replacing them entirely with new 802.11g hardware.

Fortunately, most wireless hardware (APs and client cards) in the market today gives the user the ability to upgrade the firmware (software inside ROM) of the system in order to support different technologies. Also, today's hardware has embedded microprocessors that can perform a wide variety of tasks, including IFFT and FFT, necessary for OFDM modulation.

The simulation that follows investigates the advantages that OFDM modulation would have compared to CCK in $802.11 \mathrm{~b}$ with regard to both the signal-to-noise ratio and the total speed of the wireless network. Three different simulation packages will be used to do the simulation.

\section{Simulation environment}

In order to justify the proposed use of OFDM over $802.11 \mathrm{~b}$ hardware for telemedicine applications, models have been built and tested in various simulation environments. For the lower OSI layers (physical) Matlab 6.5 and VisSim Comm 5 were used. These software packages can simulate the PHY layer very well and allow the user to introduce several variations to the communications channel like multipath noise, Rice/Rayleigh effects, selected frequency fading, etc.

For the upper OSI layers (up to the presentation layer) OPNET Modeler 9.1 was used, and allowed the opportunity to adjust data links (wired to wireless), transport sessions (the video stream will have priority over other data flowing into the network), even the presentation environment (video resolution and frame rate).

These software tools complement each other as OPNET Modeller had insufficient representation of the PHY layer and the multipath environment while Matlab and VisSim describe the lower level very effectively but fail to give the user the ability to investigate changes at higher levels. In summary, VisSim and Matlab simulation supports the proposal of using OFDM over 802.11b while OPNET Modeller investigates on the practical results that this notion would have in a telemedical application such as MedLAN.

Due to the complexity of the tasks involved, both the simulating environments mentioned above have a large number of parameters that have to be set. It would be outside the scope of this paper to mention all of them, thus only the basic settings will be included for reference purposes. 


\subsection{Physical layer simulation}

In general, the PHY layer of any IEEE 802.11 protocol that uses OFDM follows this sequence, from Data-link layer to PHY bursts:

$$
\begin{aligned}
& \text { Scrambling } \rightarrow 1 / 2 \text { rate convolutional coding } \rightarrow \\
& \text { puncturing } \rightarrow \text { interleaving } \rightarrow \text { mapping } \rightarrow \text { OFDM }
\end{aligned}
$$

Figure 4 describes how the model was built in VisSim Comm 5 (some modules were taken from Matlab): a random bit generator passes its output to a standard 802.11 scrambler. The scrambler data are input to a convolutional encoder consisting of a $1 / 2$ rate initial code and subsequent puncturing. The coded data are interleaved to avoid error bursts to be input to the convolutional decoding process. The interleaved data are subsequently mapped to data symbols (using BPSK, QPSK, etc). Finally, the output is fed into an OFDM complex modulator consisting of 48 data and four pilot subcarriers and converted using a 64-point FFT. After this, the whole train is transmitted over the air.

Figure 4 Block diagram representation of the physical layer of $802.11 \mathrm{~b}$, using OFDM modulation

802.11b OFDM Simulation Example (BPSK OFDM @ 11 Mbps)




Table 4 summarises the basic parameters of the system:

Table 4 PHY layer parameters of the model

\begin{tabular}{lc}
\hline Parameter & Value \\
\hline Modulation & BPSK, QPSK \\
Coding rate & $1 / 2$ \\
Coded bits per subcarrier & 2 \\
Coded bits per OFDM symbol & 96 \\
Data bits per OFDM symbol & 48 \\
Number of data subcarriers & 48 \\
Number of pilot subcarriers & 4 \\
FFT size & 64 \\
OFDM output rate $(11 \times 64 / 48)$ & $14.666 \mathrm{MHz}$ \\
Guard time duration & 0.8 us \\
Number of paths & 2 (multipath noise) \\
Power of additional path & $10 \%$ of original \\
\hline
\end{tabular}

Inside the AWGN box and for the single-path experiment, there is a white Gaussian noise generator with a noise level equal to the value of the EbNo box. For the multipath environment simulation, a multipath noise generator was added alongside the AWGN one. There were either one or two reflected paths. During the first scenario the reflected path had $10 \%$ of the power of the main transmission. In the second scenario, the first reflected path had $20 \%$ and the second $10 \%$ of the power of the main path.

In the demodulating side, the reverse procedure was applied. The Bit Error Rate (BER) was measured against the original signal and a power spectrum analyser displayed the original signal before it went over the noisy channel.

Overall, the transfer of a file of about $14 \mathrm{kB}$ (110 kbits) was simulated at a speed of $11 \mathrm{Mbps}$. Larger files produced the same average BER.

\subsection{Upper layer simulation}

A complete model of the MedLAN hospital environment was built using OPNET Modeller. The model represents the A\&E ward for both majors and minors, of the $\mathrm{CMH}$, West London (Figure 5). To simplify matters, only one AP was modelled, even though two (one in the majors and one in the minors) are being used in reality [2]. 
Figure 5 Model of the A\&E room of the Central Middlesex Hospital: the WLAN is installed in that ward and is connected to the rest of the hospital via the wired network

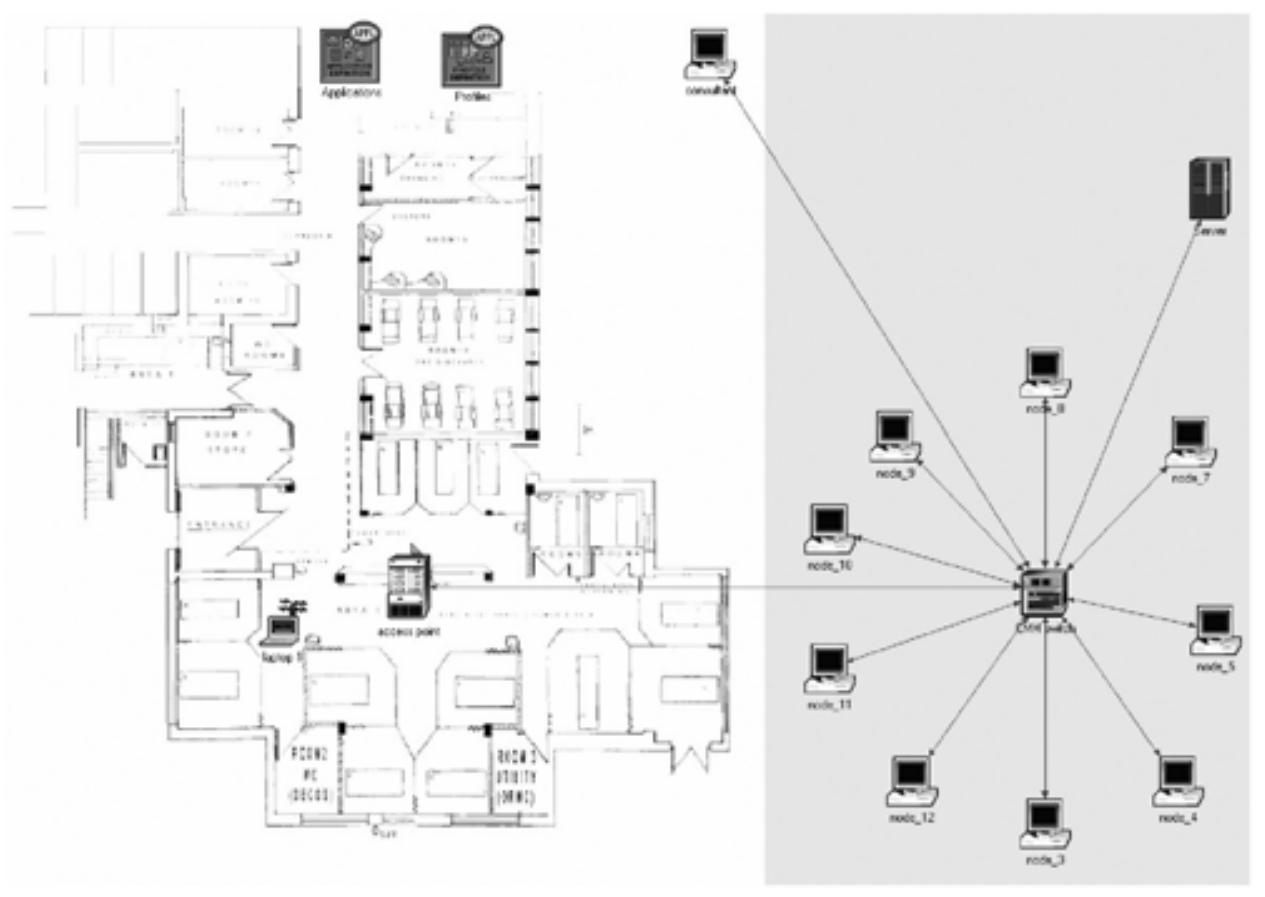

As described in Section 3, the simulated environment is a hospital requiring a wireless videoconferencing application between the A\&E department and a consultation point either within or outside the hospital. The rooms suffer from signal deterioration that can be attributed to both reflected signals due to the thickness of the walls (CMH is an old hospital) and to the existence of several noisy sources (medical equipment, microwave, etc).

The AP is located in the centre of the A\&E ward and a mobile client is free to move around the room while being in communication with the AP, which in turn is connected via wire to the hospital backbone. Several other hospital computers are also connected to the same backbone running various other applications (www browsing, e-mail, ftp, etc). All these details are described in the 'Applications' and 'Profiles' boxes of the model. One of these computers is the 'consultant' that resides within the same hospital.

The path of the mobile client was chosen to be non-static with an average distance of $25 \mathrm{~m}$ between the client and the AP. The initial 802.11 modulation is QPSK and thereafter OFDM. Power was set to a standard $30 \mathrm{~mW}$ (Cisco 340 AP). Noise levels were $5 \mathrm{~dB}$. Frames are not fragmented and no RTS/CTS commands are used as it is assumed that the mobile client would be in constant communication with the AP.

For the upper layers, a videoconferencing application was chosen having a VCR quality and a frame rate of 30 frames per second (fps). Every effort was made to keep the simulation parameters for both environments (CCK and OFDM) precisely the same. This, however, cannot be extended to every small detail like the buffer of the OFDM scenario that had to be bigger to accommodate for the FFT calculations. 
The whole model was run to simulate $300 \mathrm{~s}$ of real-time communication and took about $30 \mathrm{~min}$ to run on a P-III/850 MHz/256 MB RAM. However, the above figure depended a lot on the alternative scenarios as well as on the version of OPNET Modeller used. Finally, it is worth mentioning that the first $100 \mathrm{~s}$ had zero network traffic to allow for the networking hardware to set-up properly.

\section{Results}

For the above simulation environment, both the single-path and multipath alternatives were investigated either with standard $802.11 \mathrm{~b}$ modulation (QPSK/CCK at $11 \mathrm{Mbps}$ ), or with the addition of the OFDM modulation (QPSK/OFDM at $11 \mathrm{Mbps}$ ).

Figure 6 displays the BER vs. Eb/No of the transmitted data stream over a channel that is effected by both AWGN and multipath noise under three different modulations: QPSK/OFDM, BPSK/OFDM and QPSK/CCK, the last being the standard modulation for $802.11 \mathrm{~b}$ at $11 \mathrm{Mbps}$. It is evident that when there is no multipath noise, OFDM modulation behaves similarly with standard 802.11 b modulation (Figure 6a).

Figure $6 \mathrm{~Eb} /$ No of the signal vs. BER for various scenarios: (a) no multipath noise; just AWGN, (b) two paths (second is $10 \%$ of main path), (c) three paths (second is $20 \%$ and third $10 \%$ of original)

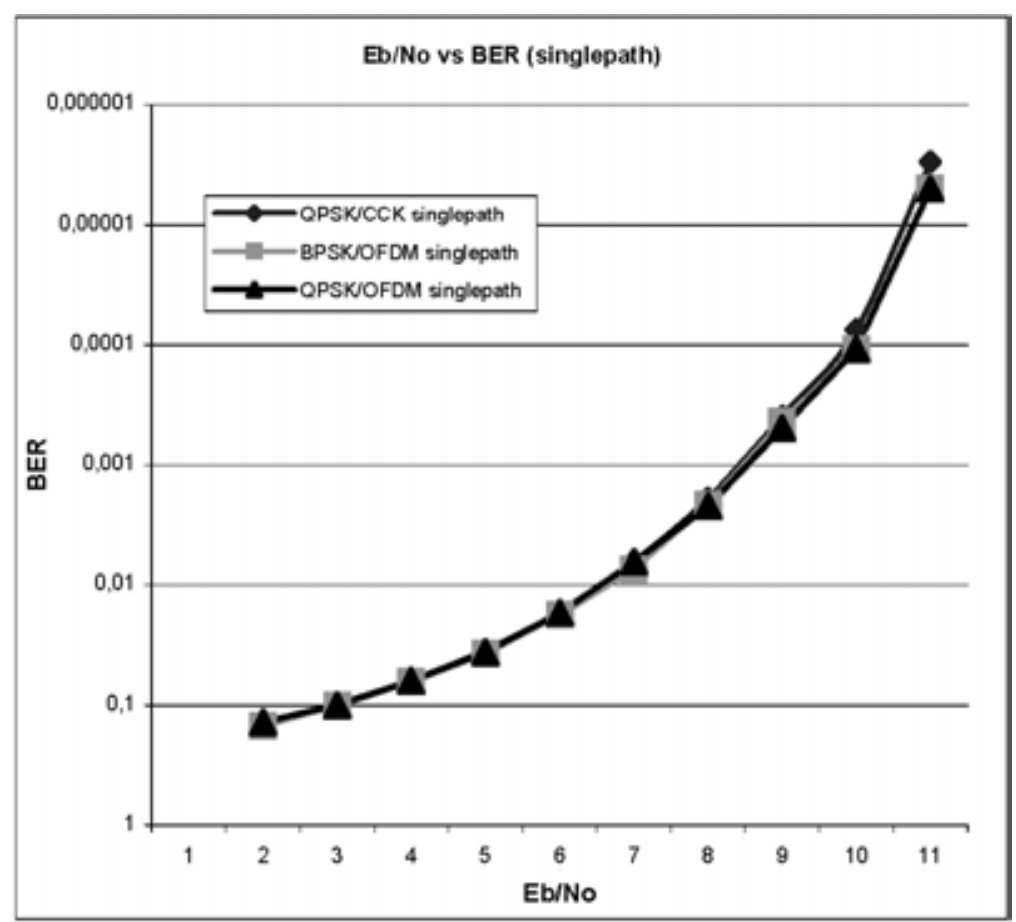

(a) 
Figure $6 \mathrm{~Eb} / \mathrm{No}$ of the signal vs. BER for various scenarios: (a) no multipath noise; just AWGN, (b) two paths (second is $10 \%$ of main path), (c) three paths (second is $20 \%$ and third $10 \%$ of original) (continued)



(b)

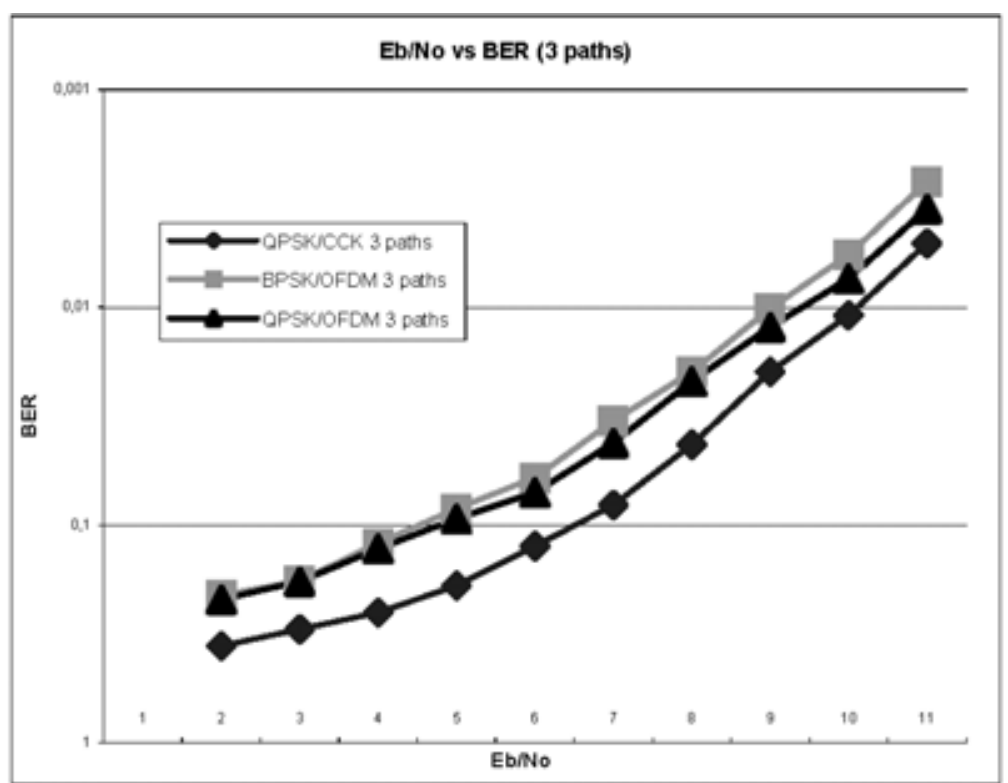

(c)

Noise values ranging from 1 to $10 \mathrm{~dB}$ were tested with $7 \mathrm{~dB}$ being a typical noise figure in an average environment. For the above values it can be seen that a clear $1 \mathrm{~dB}$ difference exists in favour of the QPSK/OFDM modulation, something that can be attributed to the more effective way that the OFDM deals with multipath interference. 
Both BPSK and QPSK behave similarly with QPSK having just a slight (but constant) advantage (Figure 6b).

As more paths were added, the gap between the CCK and OFDM modulation grew even bigger and in some cases reached up to $2 \mathrm{~dB}$ (Figure 6c). This also applies for different kinds of noise (frequency selective fading, Rice/Rayleigh effects having $250 \mathrm{~ns}$ RMS delay spread in a NLOS environment, etc) or a combination of noise sources. The average statistical error of the above measurements was $6.5 \%$.

For the upper layers that were simulated in OPNET, a great variety of statistical outputs were available, including throughput, delay, gain (dB), noise, videoconferencing, load for any of the network components (both wired and wireless) and many others. However, the throughput of the wireless client and the AP is of great importance, as it summarises many of these outputs.

Figure 7 An average increase of 1 Mbps was observed in the simulation, while using OFDM in a multipath environment

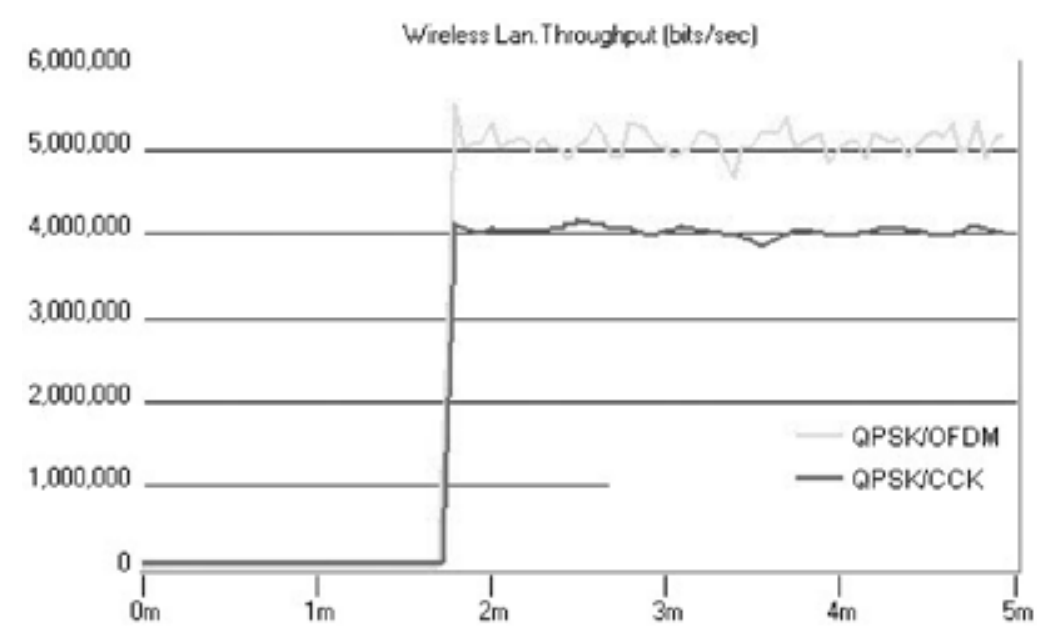

An average of $1 \mathrm{Mbps}$ improvement in the throughput was observed mainly due to the increase of Eb/No ratio described in the previous paragraph (Figure 7).

The above improvement would mean that more data can safely go through the communication channel and, for the specific telemedical application, a better video quality can be transmitted and consequently the doctors can make a more accurate diagnosis.

\section{Conclusions}

Several versions of WLANs are in the market today with IEEE 802.11a being the most recent to be commercially available in Europe. Regardless of the countries that will deregulate the $5 \mathrm{GHz}$ band (necessary to operate 802.11a), the dominance of $802.11 \mathrm{~b}$ all over Europe today is undisputable.

What this paper shows is that for specific applications like Telemedicine, the best of the two worlds can be combined. From the $802.11 \mathrm{~b}$, use the extended range it has, its increased compatibility with radio regulatory committees all over the world and its 
relatively cheap hardware. From the 802.11a, use its higher tolerance to multipath noise, a factor that affects extensively the signal quality and speed of the WLAN. The way this can be done is by replacing IEEE 802.11b's modulation with that of IEEE 802.11a, thus using OFDM modulation instead of CCK.

Since 802.11a and 802.11b do not support QoS, real-time applications are left to the mercy of the transport layer that, in turn, is highly dependent on the physical layer of the system. Real-time applications would benefit considerably by minimising the number of errors due to signals being reflected over various surfaces and decreasing the quality of the communication channel.

Telemedical applications like MedLAN could take advantage of this 'safer' communication environment and could be able to deliver a larger amount of data (in a highly reflective terrain, like an old hospital) that would result in safer and more accurate diagnosis.

To implement the above, most hardware manufacturers give end-users the ability to upgrade (flash) their firmware to include newer modulation techniques and advances at no cost and through their support webpages. Provided that these hardware manufacturers will make such alternative firmware available, it would only take hospitals a small amount of time and no cost to include OFDM with available IEEE 802.11b modulations. This offers a great advantage compared with the costly and time-consuming procedure of replacing and reconfiguring the entire WLAN with either IEEE 802 or the newer IEEE 802.11g: a compete replacement of APs is needed in the former (Figure 1), while in the latter the cost of the newly released hardware is considerable.

The above proposal has been modelled using two different simulation packages that specialise in different OSI layers. The conclusion based on the simulations is that, despite the slightly increased computational power required of the WLAN's CPUs, using OFDM modulation over IEEE 802.11 b hardware can considerably reduce the multipath noise, thus increasing the available bandwidth.

\section{References}

1 Banitsas, K.A., Istepanian, R. and Tachakra, S. (2002) 'Applications of wireless LAN systems (MedLAN)', IJMM, Vol. 2, No. 2, pp.136-142.

2 Banitsas, K.A., Tachakra, S. and Istepanian, R.H. (2002) 'Operational parameters of a medical wireless LAN: security, range and interference issues', Presented at IEEE EMBS conference 2002, Houston, Texas.

3 Siau, K. and Shen, Z. (2003) 'Mobile communications and mobile services', International Journal of Mobile Communications, Vol. 1, No 1, pp.3-14.

4 Cheung, D. and Prettie, C. (Jan 2002) 'A path loss comparison between the $5 \mathrm{GHz}$ UNII band (802.11a) and the $2.4 \mathrm{GHz}$ ISM band (802.11b)', Intel labs.

5 Flickenger, R. (26/2/03) Performance Test: 802.11b, http://www.oreillynet.com/lpt/a/713.

6 IEEE 802.11a and b specifications 15/7/03 http://standards.ieee.org/getieee802/802.11.html.

7 Laxmin Arayanan, S. and Molta, D. (2002) 'Perfect harmony', Network Computing, Vol. 66.

8 Wilson, R. (Aug 2002) 'Propagation losses through common building materials: 2.4 vs $5 \mathrm{GHz}$ ' Magis Network, August 2002.

9 ETSI TS 101475 v 1.1 .1 (2000-04) Hiperlan Type 2 Physical (PHY) Layer Technical Specifications. 
10 Khun-Jush, J., Malmgren, G., Schramm, P. and Torsner, J. (2000) 'HIPERLAN type 2 for broadband wireless communication', Ericsson Review, Vol. 2, pp.108-119.

11 Doufexi, A. and Armour, S. (May 2002) 'A comparison of the Hiperlan/2 and IEEE 802.11a wireless LAN standards', IEEE Communications Magazine, pp.172-179.

12 Ohira, H., Kodama, M. and Yoshimoto, M. (2003) 'A world first development of a multipoint videophone system over 3G-324M protocol', International Journal of Mobile Communications, Vol. 1, No 3, pp.264-272.

13 Sigurdson, J. (2003) 'New services in 3G - new business models for streaming video', International Journal of Mobile Communications, Vol. 1, Nos. 1-2, pp.15-34.

14 Owens, T., Tachakra, S., Banitsas, K.A. and Istepanian, R.H. (2001) 'Securing a medical wireless LAN System', presented at IEEE EMBC 2001, Istanbul.

15 van Nee, R. and Prasad, R. (2000) OFDM for Wireless Multimedia Communications, Artech house.

16 Keong, T.P. (6/6/03) Orthogonal Frequency Division Multiplexing (OFDM) COFDM and OFDM Bit Error Rate Simulation Using DBPSK, www.ee.mtu.edu/faculty/ztian/ee5950/ Teh_seminar.pdf.

17 Sheu, S-T., Wang, Y-D., Yin, H-C. and Chen, J. (2003) 'Adaptive rate controller for mobile ad-hoc networks', International Journal of Mobile Communications, Vol. 1, No. 3, pp.312-328.

18 Kapp, S. (Jan-Feb 2002) '802.11 Leaving the wire behind', IEEE Internet Computing, pp.82-85.

19 Kapp, S. (July-Aug 2002) '802.11a more bandwidth without the wires', IEEE Internet Computing, pp.75-79. 